Revue d'histoire de l'enfance « irrégulière »

Le Temps de l'histoire

Hors-série | 2001

Histoire et justice, panorama de la recherche

\title{
Les sacs à procès ou le travail des juges sous Louis XIII
}

\section{Christophe Blanquie}

\section{(2) OpenEdition}

\section{Journals}

Édition électronique

URL : http://journals.openedition.org/rhei/449

DOI : 10.4000/rhei.449

ISBN : 978-2-7535-1641-0

ISSN : $1777-540 \mathrm{X}$

Éditeur

Presses universitaires de Rennes

Édition imprimée

Date de publication : 15 novembre 2001

Pagination : 181-192

ISSN : 1287-2431

Référence électronique

Christophe Blanquie, "Les sacs à procès ou le travail des juges sous Louis XIII », Revue d'histoire de l'enfance « irrégulière » [En ligne], Hors-série | 2001, mis en ligne le 31 mai 2007, consulté le 19 avril 2019. URL : http://journals.openedition.org/rhei/449 ; DOI : 10.4000/rhei.449 


\section{Les sacs à procès ou le travail des juges sous Louis XIII}

La diversité de la justice de la France moderne rappelle les étapes de la formation du royaume, ${ }^{(1)}$ elle témoigne aussi de l'impact de la vénalité des offices. Outre les particularités des coutumes, chaque sénéchaussée peut posséder son style, qui définit la manière de procéder qu'on y suit, et qui s'adapte à la nature du contentieux. De surcroitt, dès lors que les officiers sont propriétaires de leurs charges et, à bien des égards, des compétences qui y sont attachées, il s'établit dans chaque corps un équilibre entre les magistrats selon la manière dont ils assemblent les diverses fonctions : ici, le lieutenant général sera lieutenant général civil et criminel ; là, il sera également président ; ailleurs il aura conservé la charge de commissaire examinateur que les conseillers d'autres sièges exercent collectivement ou à tour de rôle. Comme l'avoue Ernest Laurain, auteur de la seule étude d'ensemble consacrée aux présidiaux, " avec cette variété, il est difficile de noter dans le détail tous les droits judiciaires " du lieutenant général. ${ }^{(2)}$ Dès lors, décrire une procédure-type, définir un modèle moyen revient à s'interdire de comprendre l'organisation de chaque siège. Dans ces conditions, la tentation est d'autant plus forte de prendre acte d'une variété qui semble défier l'analyse selon laquelle l'histoire judiciaire délaisse souvent les conditions concrètes d'exercice des offices pour s'en tenir aux édits et ordonnances. Robert Chanaud présente la procédure civile d'après l'ordonnance de $1667^{(3)}$; Sylvain Soleil décrit les variations de son application selon le règlement en vigueur à Angers et assoit son analyse de la procédure criminelle sur l'ordonnance de $1670,{ }^{(4)}$ comme l'avaient fait André Laingui et Arlette Lebigre dans leur manuel. ${ }^{(5)} \mathrm{Or}$, par les règlements d'offices, ${ }^{(6)}$ le Conseil du roi, le Grand Conseil ou les parlements énoncent les fonctions respectives de chaque magistrat. De tels

\section{Christophe Blanquie}

(1) Arlette LEBIGRE,

La justice du roi,

Bruxelles, 1995 (Paris,

1988), 317 p., pp. 89-91.

(2) Ernest LAURAIN, Essai sur les présidiaux, Paris, 1896, 256 p., p. 193. Sur les attributions des officiers, voir pp. 184-230.

(3) Robert CHA-

NAUD, "Les archives des bailliages, sénéchaussées et sièges présidiaux”, Gazette des archives, 1992, n $158-159$, pp. 211-223, pp. 213-216.

(4) Sylvain SOLEIL, Le siège royal de la sénéchaussée et du présidial d’Angers (1551-1790), Rennes, Presses universitaires de Rennes, 1997, 381 p., pp. 239-245, 259-262.

(5) André LAINGUI et Arlette LEBIGRE,

Christophe Blanquie / pp. 181 à 192 
Histoire du droit pénal,

Paris, Cujas, 1980, 2 vol., t. II, "La procédure criminelle”, pp. 87-109.

(6) Sur la justice réglée, voir Philippe PAYEN, La physiologie de l'arrêt de règlement du parlement de Paris au XVIIlème siècle, Paris, PUF, 1999, 502 p., pp. 149-171.

(7) Dan. JOUSSE, Traité de la juridiction des présidiaux, Paris, Debure père, 1764, 681 p., p. $x x j$.

(8 FURTIÈRE, Le roman bourgeois, Jacq. Prévot édit., Paris, GF, 1981, 306 p., p. 172.

(9) Les présidiaux jugent en dernier ressort les causes inférieures à 250 livres ou 10 livres de rente (premier cas de l'édit), et, sous réserve d'appel au parlement, les causes comprises entre 250 et 500 livres, ou 20 livres de rente (second cas de l'édit).

(9) Voir l'article $7 \mathrm{du}$ règlement du 8 juin arrêts ne valent que pour les sièges en cause, on y trouve néanmoins "plusieurs dispositions dont on pourra faire usage pour fixer les fonctions, droits et devoirs des officiers de ces juridictions $"{ }^{(7)}$ On peut donc s'efforcer de définir l'esprit de l'institution en identifiant les points de clivage.

C'est cette approche que nous voudrions illustrer en précisant le travail des magistrats présidiaux à l'époque de Louis XIII et de Richelieu, au moment où le nombre de ces juridictions, créées par Henri II en 1552, augmente d'un tiers. Pour cela, il faut comprendre comment les procureurs constituent ces "gros dossiers qui font suer les laquais des conseillers qui vont les mettre sur le bureau ${ }^{(8)}$ " et comment les juges en prennent connaissance. C'est pourquoi nous verrons d'abord comment les parties initient les procédures. Nous suivrons ensuite le déroulement de l'audience avant de nous attacher aux procès par écrit qu'un rapporteur présente aux autres juges en la chambre du conseil.

\section{La constitution du dossier jusqu'à l'audience}

Un plaideur commence par choisir son procureur, auquel il remet les pièces qui justifient ses prétentions. La première tâche du praticien est de se rendre au greffe des présentations du présidial, où un commis reçoit la cédule de la cause, signée de sa main, et sur laquelle figurent le nom du procureur et celui des parties, ainsi que leur adresse, dont dépendent les délais entre deux étapes de la procédure. Chacun dispose en général d'une huitaine pour répondre à son adversaire s'il habite en ville, et de quinze jours s'il en est éloigné de plus de cinq à six lieues. L'enregistrement de la cédule fait courir le délai des défauts. Aussi, chaque samedi, le greffier des présentations ouvre un cahier où il note à la fois les cédules qu'il a enregistrées depuis une semaine et la date d'échéance des assignations. Le lundi, les procureurs sont tenus de se présenter au greffe. L'un d'entre eux, en signant la cédule, signale que la partie assignée a constitué procureur.

C'est au stade de l'introduction de la cause que les procureurs doivent, le cas échéant, restreindre leurs demandes aux deux cas de l'édit ${ }^{(9)}$ : l'affaire relève alors du présidial et non du sénéchal. Quelle que soit la nature sénéchale ou présidiale des causes civiles, leur instruction appar- 
tient le plus souvent au lieutenant général jusqu’à ce qu'elles soient distribuées à un rapporteur..$^{(10)}$

Ouvert pour la cédule, le sac à procès grossit vite : il comprend des copies, signées des procureurs, des pièces sur lesquelles ils fondent leurs prétentions ou leurs défenses, les répliques, dupliques et tripliques ainsi que les inventaires des productions jointes aux requêtes successives, dont ils se donnent mutuellement communication, si bien que le sac qu'ils emplissent au greffe a son équivalent dans leur étude où bien des parties ont égaré les titres des droits qu'elles entendaient défendre. Les procureurs peuvent prendre entre eux tous appointements nécessaires à la mise en état de l'affaire sans que celle-ci vienne encore en jugement. Il ne s'agit pas d'empêcher l'audience en multipliant les incidents mais de circonscrire les termes exacts du conflit "affin qu'il ne reste qu'a venir pour les advocats plaider au principal ${ }^{(11)}$ ". Ce n'est qu'à défaut d'accord sur la procédure préalable qu'ils adressent leurs requêtes au lieutenant général, lequel rendra alors un ou plusieurs jugements préparatoires. Avec son intervention, commence l'instance qui dure jusqu'à l'audience.

La première phase de la procédure repose sur les deux praticiens. Il leur appartient en effet de prendre par eux-mêmes toutes dispositions pour l'instruction des dossiers. Les règlements fournissent peut-être l'une des explications du malaise des avocats dans le premier XVIIème siècle car tandis que le rôle des procureurs se précise, certains magistrats en profitent pour essayer de marginaliser les avocats, en les empêchant soit de plaider ${ }^{(12)}$ soit de remplacer des magistrats absents. ${ }^{(13)}$

Le greffe n'intervient que pour garantir la régularité de la procédure (droits de la partie assignée, défauts n'emportant pas profit, communication des pièces...), sans quoi la mécanique judiciaire s'enraye. Ainsi, en 1642, les magistrats du présidial d'Agen déplorent que les procureurs, "par suite de leur mésintelligence à se rendre les procès, sont enfin contraints de les mettre en distribution sur de simples étiquettes et sacs vides". Aussi leur enjoignent-ils de remettre les procès au greffe, dès l'appointement en droit, "sacs remplis et pièces inventoriées ${ }^{(14)}$ ".

En revanche, le greffe assure le cas échéant la saisine du parquet. Le procureur et les avocats du roi se communiquent réciproquement les
1619 pour Moulins :

«Pareillement ledit

Lieutenant General aura

l'instruction de tous procez de l'Audience, civils et cri-

minels, incidents audit civil : décrets, interrogatoires, rapports de Jurez : ensemble des advis des parens, tutelles, curatelles, partages, inventaires, criées, descentes réparations, auditions de comptes, debats, sentences d'ordre \& de distributions de deniers et requestes, fors et exceptéles debats formez ausdits ordres qui entreront en distribution" (Joly, t. II, p. 1041). Sur l'instruction par le rapporteur, voir l'article 24 du règlement du 14 août 1617 pour Bourges (ibid., p. 1033). Toutefois, l'article $20 \mathrm{du}$ règlement rendu par le Conseil du roi pour Toulouse le 20 juin 1634 confie "l'instruction des procès présidiaux non distribués au premier magistrat sur ce requis par les parties" (Sam. Descorbiac, Recueil 
des édits, arrêts et règlements entre baillis, sénéchaux... Paris, R. Fo, et, 1638, p. 47).

(11) Arch. nat., X1a/ $2077 \mathrm{f}^{\circ} 722 \mathrm{v}^{\circ}$, règlement pour les procureurs et l'huissier audiencier de Chaumont.

(12) Voir la supplique des avocats au présidial de Sens, "contenant que par dessein prémédité par les officiers $d u d$. présidial, il se sont avisé d'interdire les "supplians" et pour y parvenir mandé les procureurs aud. siège et à eux faict deffences de charger les "supplians" d'aucunes causes à plaider ny d'aucuns procès par escript " (Arch. nat., $\left.\mathrm{X} 1 \mathrm{a} / 2147 \mathrm{f}^{\circ} 1 \mathrm{v}^{\circ}\right)$, ou la requête de cet huissier au présidial de La Rochelle, interdit pour avoir refusé d'emprisonner le syndic des avocats (Arch. nat., $\mathrm{X} 1 \mathrm{a} / 2072 \mathrm{f}^{\circ} 5 \mathrm{v}^{\circ}$ ).

(13) Arch. nat., X1a $2074 f^{\circ} 492$.

(14) Francis HABASQUE édit., Le Livre doré du présidial d'Agen, affaires introduites au siège afin de distinguer celles où le procureur du roi prendra des conclusions écrites de celles où les deux avocats du roi plaideront à tour de rôle. Dans tous les cas, la position du parquet aura été arrêtée collégialement. Dans l'immédiat, la saisine du parquet trouble le tête-à-tête des procureurs. Désormais le procureur du roi suit l'instruction. ${ }^{(15)}$ Il peut assister aux descentes, transports et verbaux ordonnés par le lieutenant général pour déterminer la matérialité des faits ou évaluer un dommage. La réalisation de ces mesures d'instruction appartient aux enquêteurs et commissaires examinateurs, dont les fonctions relevaient auparavant des lieutenants généraux. Aussi, nombre d'entre eux associent-ils les deux offices, tandis que les autres ne manquent pas d'exercer le plus souvent possible des compétences qui sont le prolongement direct des leurs. ${ }^{(16)}$ Une des meilleures garanties que les règlements puissent offrir aux détenteurs des offices de commissaires examinateurs est d'exiger que le greffe tienne registre des appointements donnés ou pris entre les parties et des jugements ordonnant une enquête. ${ }^{(17)}$ Il se révèle cependant difficile d'exclure les juges d'opérations que les parties sollicitent d'eux, de sorte que les enquêteurs interviennent alors en qualité d'adjoints. ${ }^{(18)}$

Le parquet intervenant dans les causes qui intéressent le roi ou le public, il est normal qu'il reçoive celui-ci et, si en 1603, le règlement de Bourg-en-Bresse ne réserve que deux jours par semaine à la réception des parties et de leurs conseils, ${ }^{(19)}$ celui du parquet de Saint-Pierre-leMoûtier, en 1643, invite le procureur et les avocats du roi à "s'assembler au parquet une heure avant leur audience pour recevoir les communiquations des advocats et procureurs et prendre conclusions ${ }^{(20)}$ ".

Cette phase franchie, les procureurs demandent alors l'inscription de la cause au rôle de l'audience, ce que l'huissier audiencier doit n'accorder qu'après avoir vérifié auprès du greffe la régularité de la procédure antérieure, et perçu les droits conséquents. ${ }^{(21)}$ Lorsque les affaires ne supposent pas d'instruction, un procureur peut se contenter d'avertir la partie adverse qu'il présentera un placet à la prochaine audience. L'officier qui préside conserve toujours la faculté d'appeler, en sus du rôle, une poignée de placets. 


\section{L'audience}

Il faut se représenter le palais de justice aux premières lueurs du jour. Devant la porte, piétinent des plaideurs, leurs procureurs et leurs avocats. Les procureurs s'assurent que leurs causes sont prêtes à être plaidées. Pendant ce temps, les premiers juges arrivent, accompagnés d'un valet, qui les éclaire. Survient l'un des principaux officiers du siège, précédé de deux huissiers, leur bâton à la main. Il gagne la chambre du conseil où il vérifie le rôle de l'audience et s'accorde avec les conseillers qui vont l'accompagner à l'auditoire, tandis que le procureur et les avocats du roi, assemblés en leur parquet, s'accordent sur les affaires du jour. Avant de rendre la justice, les officiers vont écouter la messe dans la chapelle du palais. Après une heure d'attente, l'huissier de semaine ouvre la porte de l'auditoire et laisse entrer procureurs et avocats qui vont prendre place sur leurs bancs, derrière la barre qui isole les magistrats du public ; il s'assure aussi que les parties sont décemment vêtues et filtre les curieux.

Les magistrats viennent prendre séance. Il faut ici distinguer la France des bailliages, où tous les conseillers montent à l'audience, et celle des sénéchaussées, dans lesquelles le lieutenant général la tient seul et conserve la faculté d'y appeler les conseillers de son choix. Lui-même juge mage, Samuel Descorbiac le souligne avec force, le règlement du 31 mars 1620 pour Villefranche-de-Rouergue confirme que "le JugeMage ne sera poinct abstraint de prendre des Conseillers à l'Audiance de l'ordinaire ${ }^{(22)}$ ». Toutefois, les conseillers obtiennent quelques aménagements et l'arrêt autorise le juge mage à s'entourer de quatre d'entre eux à l'audience de l'ordinaire.

En revanche, la situation apparaît plus nuancée dans le vaste ressort du parlement de Paris, si l'on en croit l'arrêt rendu pour Limoges par les Requêtes de l'Hôtel en 1637 : "les audiences de la juridiction ordinaire du sénéchal se tiendront par le lieutenant général seul et continuera à l'avenir aux jours de mardy et jeudy depuis sept jusques àbuict heures pour les affaires légères et sommaires, et après ladicte heure passée, les autres causes importantes seront plaidées, à laquelle audience de buict heures les conseillers du siège pourront assister sy bon leur semble. Et prendra leur advis et prononcera à la pluralité des voix ${ }^{(23)}$ ".
Archives historiques du département de la Gironde, t. 42, ParisBordeaux, 1907, 548 p., p. 17.

(15) Voir par exemple, Arch. nat., X1a/2173/b f 139 .

(16) Arch. nat., X1a/ $2023 f^{\circ} 29$, règlement pour les commissaires examinateurs de Lyon.

(17) Arch. nat., X1a/ $2183 \mathrm{f}^{\circ} 94$, règlement pour les enquêteurs de Chaumont.

(18) Arch. nat., X1a/ $2029 \mathrm{f}^{\circ} 615$, règlement pour les enquêteurs et commissaires examinateurs du Mans.

(19) Dan. JOUSSE, op. cit., p. 50.

(20) Arch. nat., X1a/ $2187 \mathrm{f}^{\circ} 24$

(21) Arch. nat., X1a/ 2023 f $^{\circ} 230$.

(22) Sam. Descorbiac, op. cit., p. 67. On appelle juges mages les lieutenants généraux de la France méridionale.

(23) Arch. nat., V 4 108, 9 janvier 1637. 
(24) Arch. nat., V 4 116, 23 octobre 1638.
L'assistance des conseillers à l'audience constitue déjà un droit lorsque leurs homologues des sièges du ressort de Toulouse et de Bordeaux doivent encore être priés par les lieutenants généraux et juges mages. La question rebondit toutefois dès le jugement rendu, aussi les Requêtes de l'Hôtel sont-elles invitées à préciser de nouveau les droits du lieutenant général. Le 23 octobre 1638, un nouveau règlement vient confirmer les modalités de l'audience ordinaire : "en la petite audiance ordinaire du Seneschal qui se tient depuis sept heures jusques àbuict heures, seront jugées les causes sommaires \& légères, savoir preparatoires, forclusions et autres instructions de procès civils de la juridiction du Seneschal, les causes de police, main levée de menbles, saisie ou executez $\sigma$ autres causes privilégiées qui requierent célérité ». Tandis que l'arrêt ne prévoit pas que le lieutenant général puisse inviter les conseillers à participer à siéger à la petite audience, il dispose que si ceux-ci y entraient, le lieutenant général ne sera pas tenu de prendre leurs avis si bon ne lui semble. ${ }^{(24)}$

La différence se marque par le fait que les avocats s'adressent alors au seul lieutenant général et non à tous les magistrats. Dans tous les cas, l'audience se décompose en deux parties, l'une réservée aux jugements préparatoires, l'autre aux jugements sur le fond.

L'huissier audiencier, le rôle à la main, appelle la première affaire que les avocats plaident. Une fois que le parquet a été ouï, le lieutenant général pose quelques questions jusqu'à ce qu'il estime l'affaire suffisamment claire. S'il juge seul, il prononce immédiatement la sentence ; sinon, il prend avis des juges qui l'assistent et qui opinent à tour de rôle, certains invitant d'abord le lieutenant général à demander une précision supplémentaire. Puis le lieutenant général prononce à la pluralité des voix. L'affaire peut être jugée sur le champ, mais il arrive aussi que les magistrats requièrent un justificatif, une explication : l'affaire sera alors vidée sur le registre par l'un des magistrats ayant siégé à l'audience. Enfin, quelques affaires sont trop complexes pour être tranchées aussi rapidement : elles appellent un examen plus complet, qui sera éclairé par le rapport d'un conseiller. Elles seront alors appointées pour être jugées par écrit. Dans ce cas, les magistrats ne débattent pas du fond ; ils opinent seulement sur l'opportunité d'appointer, une minorité qualifiée 
suffisant pour emporter la décision. L'appointement en droit n'est pas réservé aux sacs à procès les plus volumineux et l'on appointe aussi des monocules, c'est-à-dire des sacs qui comportent une seule pièce.

Une audience ne dure que deux heures. Les débats sont assez rapides : en cas de difficulté, les affaires sont soit renvoyées à une audience ultérieure, soit appointées au conseil. Quand les procureurs postulants de Saint-Pierre-le-Moûtier se plaignent que les magistrats ne distinguent pas les jugements préparatoires des définitifs, de sorte qu'il y a plus d'une centaine de prononciations par audience, ${ }^{(25)}$ ils montrent l'importance des règlements qui, en énonçant les jours et les heures des différentes audiences, permettent d'expédier plus rapidement les causes et facilitent le travail des auxiliaires de justice. Cette considération technique rejoint une contrainte pratique : dans de nombreux sièges, le palais de justice se réduit à deux salles, d'abord un auditoire, que les magistrats partagent souvent avec d'autres juges et où ils doivent tenir séparément les audiences civiles et criminelles, sénéchales et présidiales, une chambre du conseil ensuite.

A la fin de l'audience, le lieutenant général descend de sa chaire et s'approche de la barre pour les issues; il va trancher quelques affaires sommaires et dont le montant n'excède pas 10 livres : ne nécessitant qu'une procédure allégée, elles sont expédiées à l'issue de l'audience. ${ }^{(26)}$ Tandis que le lieutenant général quitte l'auditoire, le commis du greffier met en ordre le plumitif qu'il lui fera ensuite signer. Ceux des magistrats qui ne profitent pas de la buvette du palais ${ }^{(27)}$ se hâtent alors de gagner leur hôtel pour le repas de midi, car après dîner, ils reprendront le chemin du tribunal, accompagnés de leur secrétaire ou d'un laquais chargé des sacs des procès par écrit dont ils ont préparé les rapports.

Le déroulement des audiences présidiales est similaire. Cependant, un président présidial les préside et les magistrats, parmi lesquels peuvent siéger des magistrats honoraires, doivent être au moins sept. Faute de quorum, on fait appel à des avocats, qui prendront part aux émoluments. A la différence du lieutenant général, le président présidial ne suit pas la mise en état des affaires. Il se borne à présider et à prononcer les jugements à la pluralité des voix. La comparaison entre les deux chefs
(25) Arch. nat., X1a/ 2093 f $^{\circ} 424$.

(26) Les issues correspondent à la petite audience que le lieutenant général de Limoges tient à l'ouverture du siège, avant que les conseillers ne le rejoignent.

(27) Évoquant la mémoire de sa femme, Perrin Dandin soupire :

« Elle ê̂t du buvetier emporté les serviettes/Plutôt que de rentrer au logis les mains nettes» (I, 4). 
(28) André Joubert, La bibliothèque et le mobilier d'un lieutenant particulier au siège royal de ChasteauGontier sous Louis XIII (1626-1627), G. Fleury et Dangin, 39 p., Mamers, 1888. de la juridiction rappelle que la juridiction du sénéchal relève du lieutenant général alors que celle du présidial appartient en corps aux magistrats du siège.

Toutes les semaines ou tous les quinze jours, selon l'affluence des causes, le samedi après-midi, le greffier apporte au chef de la juridiction la liste des procès appointés au conseil, afin qu'il les distribue. On retrouve ici de profondes différences entre les sièges. Dans certains cas, la distribution est confiée au président présidial, dans d'autres, au lieutenant général, ailleurs encore le premier distribue les affaires des deux cas de l'édit, et le second celles de l'ordinaire. Partout, deux commissaires assistent à la distribution, sans jamais y avoir voix délibérative mais avec parfois le droit de choisir un rapport après que le lieutenant général a été servi de son préciput et à son tour, et avant qu'on ne serve les autres conseillers, toujours "eu égard à leur mérite et à leur ancienneté et à la qualité des matières”. Celui qui procède à la distribution doit s'efforcer d'assigner aux juges les procès les plus complexes à des juges chevronnés sans trop désavantager les plus jeunes. L'une des méthodes utilisées consiste, comme à Nîmes, à classer les procès selon leur importance avant de distribuer, catégorie par catégorie.

\section{Le travail du rapporteur}

Lorsqu'un procès lui a été distribué, le magistrat en retire le sac au greffe (le greffier lui fait signer un registre, en guise de décharge), et l'emporte chez lui. Car c'est en son hôtel et non au palais, qu'il étudie les pièces et procède aux recherches nécessaires parmi les volumes de sa bibliothèque. L'inventaire de celle de René Quantin, lieutenant particulier à Château-Gontier, emporté par une épidémie en 1626, signale 103 titres, parmi lesquels on relève aussi bien des ouvrages de pratique, ainsi Les trois notaires de Papon ou le Commentaire de la coutume d'Anjou par Chopin, que la toute récente Irénarchie de Jean d'Arrerac. C'est aussi parce que les sacs à procès dont il a le rapport y voisinent avec ses papiers de famille que l'inventaire est mené par un représentant du lieutenant général accompagné du greffier. ${ }^{(28)}$ De fait, le juge exerce son activité professionnelle chez lui au point que cela influence la pratique de 
ses affaires familiales. ${ }^{(29)}$ Point de rupture non plus entre les méthodes utilisées pour étudier les sacs à procès ou pour composer les ouvrages d'histoire auxquels se consacrent plusieurs magistrats présidiaux. ${ }^{(30)}$

On comprend alors pourquoi c'est à leur domicile que les plaideurs viennent solliciter les juges : c'est là que s'achève la mise en état de leur affaire et que s'en prépare le jugement. Contrairement à une idée reçue, l'usage, s'il survit à son interdiction, répond à des contraintes concrètes, au point que le règlement de Chaumont impose aux procureurs de se présenter au rapporteur dès le lundi qui suit la distribution. Le rapporteur est d'ailleurs compétent pour ordonner les mesures d'instruction encore nécessaires : une fois le procès distribué, il peut prendre les décisions qui relevaient auparavant du lieutenant général.

De surcroît, le lieutenant général et, en son absence, les autres magistrats du siège, peuvent expédier chez eux ce qui relève de la juridiction volontaire, et qui intéresse essentiellement le droit de la famille. L'un des reproches que les conseillers lui adressent le plus fréquemment est de rendre la justice en leur domicile. ${ }^{(31)}$ La présence du procureur du roi tend à limiter les abus mais les avocats du roi ne manquent pas d'incriminer leur collègue du parquet ${ }^{(32)}$ et la présence du greffier devient alors une garantie supplémentaire.

Cette pratique explique le regroupement des demeures des magistrats à proximité du palais de justice et la superposition de rapports de sociabilité à ceux qui s'établissent pour l'expédition des causes. De la même manière, ces allées et venues entre l'auditoire, où ils rendent la justice, et l'étude, où ils reçoivent les justiciables, justifient que les magistrats conservent partout la tenue décente qui convient à leur dignité : l'obligation de porter la soutane et le bonnet carréne ne s'applique pas seulement au palais, même si elle connaît quelques atténuations hors de la ville, pendant les vacations.

Le juge doit dépouiller la totalité des sacs à procès, en préparer un résumé analytique et proposer un projet d'arrêt. Le rapporteur établit de sa main des "extraits" qui présentent la substance des faits, telle qu'elle résulte des pièces produites et de manière à faire apparaître les points de droit sur lesquels il faut prononcer. L'extrait préfigure les visas qui figu-
(29) Éric TUNC,

"Stratégies matrimoniales et espaces", Cabiers du centre de recherches historiques, octobre 1999, pp. 103119, pp. 117-118.

(30) Michel CASSAN, "Les Antiquités d'Agen par Jean Darnalt", Monluc, d’Aubigné. Deux épées, deux plumes, Jean Cubelier de Beynac et Cl.-Gilb. Dubois édit., Agen, 1999, pp. 27-43, p. 38.

(31) Ainsi la requête du lieutenant particulier de Provins, le 17 mai 1632 , pour que « deffences fussent faictes audict Boisard [lieutenant général] et aultres officiers dudit siège de donner aulcunes assignations aux parties en leurs domicilles, ny y rendre aulcunes sentences et jugemens à peyne de nullité » (Arch. nat., X1a/2053 $\left.f^{\circ} 446 \mathrm{v}^{\circ}\right)$.

(32) Ainsi à Blois en 1640 (Arch. nat., X1a/ 2152, f $\mathrm{f}^{\circ} 272$ ). 
(33) Règlement de

Bourg-en-Bresse, 24/05/1603, art. XXXVIII. L'art. VII du règlement pour Toulouse du 09/03/1575 enjoint au rapporteur d'écrire l'extrait de sa main.

(34) Xavier Martin,

"L'édition du commentaire de Dupineau sur la coutume d'Anjou (1698)", Revue française d'histoire du livre, 1989, $n^{\circ}$ 62-63, pp. 55-92, p. 72. Sur la page de titre de l'édition reproduite p. 78, on lit « $C e$ livre est pour la Chambre $d u$ Conseil de Messieurs du présidial d'Angers et a esté donné par Monsieur Boyslève de Grimand qui a pris soin de l'impression $d u$ présent livre et en a fait la depence."

(35) Christophe

Blanquie, "Le registre secret du présidial de Libourne", Revue bistorique et archéologique $d u$ Libournais, t. LXVI $\mathrm{n}^{\circ} 252$, 2ème trimestre 1999, pp. 49-58. reront dans l'arrêt, sa rédaction est conçue pour que "s'il est question de retourner auxdits faits \& relire lesdits titres ou dépositions en opinant, l'on puisse recourir audit extrait denuement vérifié, sans nécessité de relire lesdites enquêtes et autres pièces ${ }^{(33)}$ ". Dans ces causes, apparues trop complexes pour être jugées à l'audience, il appartient au rapporteur de préparer la délibération des autres magistrats et de leur soumettre une solution. Toutefois, si lors du délibéré, l'un des conseillers soulève une question de droit qui requiert de nouvelles recherches, l'affaire est renvoyée à une séance ultérieure afin que chacun puisse, chez lui, procéder aux vérifications nécessaires. Les volumes conservés dans la chambre du conseil sont des usuels, tels que le Commentaire sur la coutume d'Anjou par Dupineau, que ses successeurs au présidial d'Angers ont contribué à éditer. ${ }^{(34)}$ Le palais est un lieu de délibération, c'est l'endroit où les magistrats disent le droit mais ce n'est pas là qu'ils procèdent à l'examen détaillé des dossiers.

De même que la sénéchale, la justice présidiale est conçue comme une justice rapide et les délais de rapport sont, théoriquement, brefs : l'affaire n'est appointée pour être distribuée que lorsqu'elle semble mise en état. Les rapporteurs ne disposent que de quelques jours pour préparer leur extrait, certains règlements fixant expressément un terme de trois jours. En tout état de cause, la norme se veut inférieure au délai qui sépare deux distributions, soit, dans la majorité des cas, une quinzaine de jours et il y a des règlements pour disposer que même les conseillers qui s'absentent pour plus d'une semaine, doivent rendre les procès qui leur ont été distribués.

Les magistrats examinent les procès par écrit dans la chambre du conseil. Ils passent là bien plus de temps qu'à l'audience, car c'est également dans cette pièce qu'ils délibèrent des affaires de la compagnie et reçoivent certains officiers. C'est pourquoi il peut arriver que de telles délibérations soient mêlées aux sentences. ${ }^{(35)}$ Les règlements obligent progressivement les magistrats à distinguer les audiences du présidial et de l'ordinaire. Cette prescription, tardivement appliquée dans des sièges aussi importants qu'Orléans ou Reims, semble ignorée dans la chambre du conseil, où le président et le lieutenant général occupent chacun une place d'honneur. 
Le lieutenant général commence par présenter ses rapports, puis celui qui préside donnera le bureau à chaque rapporteur successivement. Le rapporteur "prend" le bureau, sur lequel il dépose, ou fait déposer, les sacs à procès. Chacun doit présenter succinctement toutes les pièces puis résumer l'affaire. Celui qui préside prend alors les opinions et le jugement est arrêté à la pluralité des voix. Nul doute que la majorité se rallie le plus souvent à l'avis du rapporteur ; il arrive pourtant que certaines affaires suscitent de longs débats, qu'enveniment parfois les querelles entre officiers. La délibération sur les procès par écrit n'est pas publique mais les parties peuvent y être appelées. Tant que les juges y siégeront, l'huissier de semaine reste à la porte de la salle. A tout moment, il peut recevoir l'ordre d'introduire les magistrats du parquet ou les avocats des parties qui pourront alors présenter les informations requises. C'est pourquoi un règlement pour la sénéchaussée de Bigorre rappelle que les avocats "n'entreront au Conseil lorsqu'ils y seront appelés, sans leurs robes loungues, à peyne de l'esmande ${ }^{(36)}$ ».

De telles dispositions suggèrent que les séances en la chambre du conseil peuvent être plus longues que les audiences. Tous les officiers y sont appelés à délibérer sur chaque affaire et c'est là que les nouveaux magistrats apprennent de leurs aînés à faire leur charge, comme les jeunes avocats se forment en assistant aux audiences. Leur apprentissage s'appuie sur des copies de commentaires des coutumes et des manuscrits de jurisprudence, qui assurent la célébrité des précédents et façonnent la tradition du siège.

Cette délibération collective est l'essence de l'institution présidiale, qui consiste à assurer aux moindres affaires un traitement aussi sûr que celui des parlements. Ce n'est pas que les petites juridictions ne traitent pas par écrit les affaires complexes, mais plutôt que dans les sièges importants, les toutes petites affaires relèvent de celui qui tient l'audience, les affaires simples sont expédiées avec l'avis de plusieurs magistrats et les affaires complexes sont délibérées en la chambre du conseil : l’idée n'est pas que la complexité requiert un examen écrit mais surtout qu'une affaire importante requiert les lumières d'une pluralité de magistrats.

La dernière étape du jugement concerne les dépens qu'il s'agit de taxer et d'attribuer. La taxation appartient à celui qui préside, mais le retour
(36) M. Lanore,

Inventaire sommaire des archives départementales antérieures à 1790 . HautesPyrénées, séries A et B, t. I, Tarbes, 1904, p. XI. 
d'épices similaires indique que l'on calcule en fonction d'un barème admis de tous. Le président résume alors les opinions et formule le jugement, dont le rapporteur devra rédiger le dicton ("il sera dit que ») de sa main et, après en avoir donné lecture en la chambre du conseil, le transmettre dans les trois jours au greffier, duquel les parties le retireront après avoir réglé les épices, c'est-à-dire les émoluments des officiers. Qu'elles soient ensuite partagées entre les officiers, conservées dans une bourse commune ou acquises au rapporteur, les épices sont réglées au receveur des épices qui les répartit ensuite selon les règles en vigueur dans le siège.

A suivre de la sorte les sacs à procès, on constate que l'audience publique, avec ses deux petites heures, ne revêt qu'une importance relative, d'abord parce que c'est chez eux que les rapporteurs accomplissent l'essentiel de leur mission, ensuite parce que la délibération collective intervient plutôt en la chambre du conseil qu'en l'auditoire. Le calendrier des audiences, en revanche, constitue un précieux indice de la nature du contentieux et des conditions dans lesquelles s'établit l'équilibre du corps. Le travail des magistrats se décompose en deux temps. Il y a d'abord l'instruction de l'affaire, qui appartient au lieutenant général ou à son remplaçant, et dont les autres officiers sont, au pire, les témoins et, au mieux, de simples collaborateurs, puis, après la distribution, vient la préparation du rapport en la chambre du conseil. L'esprit de l'institution présidiale est que la plupart des magistrats opinent sur la quasi-totalité des affaires et n'en rapportent qu'une faible proportion. Par conséquent, la question de l'assiduité des officiers se pose en des termes très particuliers, d'autant qu'il faut compter sur les modalités de partage des émoluments, qui varient selon la composition de la compagnie et le volume du contentieux. En revanche, toute cette organisation, où les juges sont capables de mobiliser leur science du droit pour opiner sur le champ dans des affaires qu'ils découvrent, est cohérente avec des délais de jugement plus brefs que ne l'affirme une tradition plus littéraire qu'historique ou juridique. 